\title{
Traduire
}

Une eutre perspective sur $r$ tatadciction

Revue française de la traduction

$221 \mid 2009$

Voies de l'interprétation

\section{Entretien avec Massoumeh Lahidji, interprète pour le cinéma}

Vanessa De Pizzol

\section{(2) OpenEdition}

Journals

Édition électronique

URL : http://journals.openedition.org/traduire/346

DOI : $10.4000 /$ traduire.346

ISSN : 2272-9992

Éditeur

Société française des traducteurs

Édition imprimée

Date de publication : 15 décembre 2009

Pagination : 48-57

ISSN : 0395-773X

Référence électronique

Vanessa De Pizzol, « Entretien avec Massoumeh Lahidji, interprète pour le cinéma », Traduire [En ligne], 221 | 2009, mis en ligne le 12 novembre 2013, consulté le 25 novembre 2020. URL : http:// journals.openedition.org/traduire/346 ; DOI : https://doi.org/10.4000/traduire.346 


\section{Entretien avec Massoumeh Lahidji, interprète pour le cinéma}

\section{Propos recueillis par Vanessa De Pizzol}

- Massoumeh Lahidji, vous êtes membre de l'ATAA, votre nom est associé depuis quelques années au cinéma et l'on vous voit aux côtés de metteurs en scène mettre votre talent d'interprète au service de leur parole de créateur. Votre parcours a-t-il dès le début été conditionné par cette spécialisation dans le septième art?

Le goût de la langue et des langues m'habite depuis mon plus jeune âge. Je suis née en Iran et j'allais dans une école française. Je me souviens d'avoir éprouvé dès l'école primaire la frustration de ne parler que deux langues : le persan et le français. J'étais fascinée par mes amis qui parlaient chez eux le turc ou l'arménien et j'essayais de capter leurs mots, leurs accents. Au lycée, à Paris, j'ai appris toutes les langues que le système éducatif me proposait. J'ai donc su très tôt que mon métier serait lié à cette passion. Mes parents étaient persuadés - comme beaucoup - que les langues n'étaient qu'un moyen et que je devais mettre mon aisance d'expression au service d'un métier "sérieux " tel que celui d'avocat. Mais pour moi, les langues étaient une fin, un objet d'étude, de réflexion et d'amour, en soi. J'ai donc fait des études de langues, en espagnol et en anglais, puis des études de linguistique générale, pour pousser encore plus loin cette exploration. Après un DEA de sociolinguistique, je me suis engagée, presque à mon insu, dans une carrière d'enseignement des langues en formation professionnelle, puis à l'université. J'ai beaucoup travaillé pour la fonction publique, aidant les fonctionnaires à préparer des concours administratifs. Cet univers était loin de moi et pourtant, j'ai beaucoup appris de mes élèves et je crois que j'ai réussi à faire parler et même aimer l'anglais à des adultes restés hermétiques à des années d'enseignement des langues au lycée et à l'université. Par ailleurs, la traduction était bien sûr une pratique qui faisait ma joie depuis toujours. Fidèle à la tradition française, c'est vraiment ainsi que j'ai appris les langues, en faisant des thèmes et des versions, encore plus souvent que ne me l'imposaient mes épreuves scolaires ou universitaires. J'avais fait un DESS, lors duquel il m'avait paru évident que j'avais une aptitude et un goût supérieurs pour l'interprétation. Mais je n'ai pas tenté de devenir interprète de conférence car je n'avais pas le moindre intérêt ni pour les organismes internationaux, ni pour le monde de l'entreprise! L'exercice me passionnait mais ce qui ne me 
ressemblait guère dans ce métier, c'était la capacité de s'intéresser à tout : aujourd'hui la finance, demain la géologie... Le seul champ qui m'intéressait était celui - assez vaste en soi - de la culture. Après quelques années d'enseignement, j'ai décidé de changer de cap. Mon autre passion est le cinéma. En tant que spectatrice, je réfléchis de longue date à la question de la langue au cinéma. J'ai donc décidé de faire un Master 2 de sous-titrage, pour me lancer dans ce métier et mener parallèlement une recherche sur ce sujet, peut-être dans le cadre d'une thèse de doctorat.

Pendant cette année d'études, j'ai travaillé pour un festival de cinéma, où l'on m'a demandé, tout à fait par hasard, de monter sur scène pour traduire les propos d'un cinéaste. C'était il y a un peu plus de trois ans, et depuis, c'est devenu mon activité principale !

- Votre parcours et la manière dont vous pratiquez l'interprétation déjouent fortement l'image formatée de l'interprète qui peut circuler parmi ceux qui ne connaissent pas bien la profession. Vous parliez de l'interprète de conférence, une spécialisation que vous avez exclue de votre carrière, semblant privilégier au contraire une sorte de liberté...

Quel que soit le métier que j'exerce, je n'ai jamais le sentiment de rentrer tout à fait dans le moule existant. J'ai enseigné en ressentant souvent cette impression d'usurpation. Je ne me destinais pas à ce métier, je n'avais pas suivi la voie classique pour être enseignante et pourtant, j'avais inventé ma façon de le faire, inspirée de ma formation de linguiste, de mon expérience de locutrice et d'apprenante. Une méthode qui convenait à mes étudiants, à moi-même, mais aussi à l'institution! Aujourd'hui, j'exerce le métier d'interprète sans avoir été formée par une école spécialisée et je dois avouer que les compliments qui me touchent le plus lorsque des membres du public viennent me voir après une intervention, ce sont ceux qui viennent d'interprète de conférence ! Leur reconnaissance me procure un bref sentiment de légitimité. Je me sens en effet tout à fait libre, dans la mesure où je ne travaille que dans ce champ que j'affectionne - essentiellement le cinéma, mais aussi la littérature et l'art - et selon des techniques peu orthodoxes. Je travaille presque exclusivement en consécutive et je ne prends pas de notes.

- Pour être un bon interprète, n'y a-t-il pas par conséquent quelque chose qui se trouverait au-delà de la langue, de la maîtrise théorique et orale acquise par la formation dans les écoles spécialisées et les séjours linguistiques ou tout simplement le contexte familial (langue maternelle)?

Le métier requiert des qualités variées. La maîtrise des langues et une aisance d'expression, cela va de soi. Mais il me semble qu'il faut également une capacité de concentration extrême. II s'agit de focaliser toute son attention sur un discours. C'est en fait avant tout un exercice d'écoute. Pour ma part, ce processus est dans une certaine mesure inconscient. Comme je le disais, je ne prends pas de notes lorsque j'écoute. Pour moi, la prise de notes constituerait une perte de concentration. Ma démarche consiste à laisser le discours de l'autre se déposer dans 
mon esprit, puis à le reproduire dans la langue cible dans un certain lâcher prise. Je suis moimême gênée de parler de cet exercice comme de quelque chose d'irrationnel, de magique. Cela entre en contradiction totale avec ma vision très rationnelle et très descriptive de la question linguistique, issue de ma formation de linguiste structuraliste. Je considère la langue comme un matériau très précis, que l'on peut décrire, mais aussi reproduire, selon des critères rigoureux, scientifiques. J'ai certes une technique, du reste assez proche de celle enseignée pour l'interprétation consécutive, qui consiste à dégager du discours émis des groupes d'idées, représentés par des mots-clés. Seulement, ces mots-clés, au lieu de les écrire, je les retiens dans ma mémoire. Et, au moment de la restitution, il se produit en moi une sorte d'état second, où je vis une libération de la parole. On m'a souvent fait remarquer que j'adopte jusqu'au phrasé, jusqu'à la respiration de celui que j'interprète, dans ses pauses, ses hésitations. Je ne maîtrise pas cela mais je sais que lorsqu'on travaille avec des artistes, la dimension primordiale est celle de la part subjective, personnelle, intime, du discours. II y a certes un contenu d'information dénotatif, à transmettre avec rigueur et précision, mais il y a aussi et surtout toute la dimension connotative. C'est là que l'interprète prend part au processus créatif, dans sa mise en discours. Vous devez vous couler dans l'univers mental de celui que vous interprétez. Vous devez partager et construire avec lui un instant, où une parole est véhiculée, une information, une émotion transmise. Pour avoir cette disponibilité, cette écoute concentrée, il faut être très bien préparé. Bien sûr, se sentir en pleine maîtrise des deux langues en présence mais aussi du sujet. C'est un travail d'improvisation totale. Vous ne savez jamais ce qu'un réalisateur - parfois facétieux - va répondre à une question qu'on lui pose. II faut aimer l'imprévu et s'y adapter. Ce que j'aime dans ce métier, c'est la valeur de l'instant. Vous devez donner le meilleur de vous-même dans la seconde où les choses se présentent. Trouver la formule adéquate une fois que vous êtes sortie de scène ne sert à rien! Je trouve cet enjeu jubilatoire. Reste à trouver le parfait équilibre entre la concentration et la détente.

- Vous avez évoqué l'imprégnation nécessaire de l'univers de la personne dont vous allez traduire le discours, la nécessaire connaissance de son "domaine de spécialité " sans oublier la maîtrise des langues en jeu. Mais y a-t-il pour l'interprète des gammes, comme pour le musicien, lui permettant de se maintenir, voire de s'améliorer, entre deux missions ?

Je me pose constamment cette question. Comment s'améliorer ? Comment dépasser ce qui semble acquis dans l'exercice de mon métier? Cela me préoccupe d'autant plus que de nombreuses personnes tendent à percevoir ma pratique d'interprète comme un don. Les félicitations que je reçois parfois s'apparentent davantage à celles que l'on adresse à une force de la nature qu'à un chirurgien ou un à mathématicien ! II y a bien sûr la possibilité d'élargir mon domaine de compétence au-delà du cinéma ou même de la culture mais encore une fois, je suis trop bornée pour cela. Je pourrais m'entraîner davantage pour le travail en cabine. L'interprétation simultanée, que j'ai peu pratiquée, me paraît être un exercice moins gratifiant, où vous êtes privée de la marge de liberté minimale qui vous permet de vous approprier le discours 
de l'autre et de le reformuler. Vous devez coller à l'énoncé source et j'ai tendance à vivre cela comme une contrainte. Encore plus de liberté ! Une dimension qui est très présente dans mon rapport à mon travail est celle de la réflexion métalinguistique sur le processus qui m'occupe. Je ne suis pas certaine que cela me permette de m'améliorer dans la pratique de l'exercice mais cela est vital pour ma satisfaction intellectuelle.

Pour l'exercice lui-même, il me semble que les meilleures gammes sont la pratique régulière et variée du métier. Je suis amenée à travailler dans des contextes et aux côtés de personnalités très différentes et cela est le meilleur moyen de se remettre en question et s'adapter à une nouvelle situation à chaque fois, de ne pas s'endormir sur ses lauriers.

- Dans votre démarche d'interprète, votre implication vis-à-vis de la personne dont vous traduisez les propos semble primordiale. Comment décririez-vous cette relation?

II s'agit d'un rapport de confiance. Vous avez un artiste qui vient parler de lui, de son œuvre, du film qui l'occupe depuis des mois, voire des années. Il est seul, face à une assemblée qui ne parle pas sa langue. II vous confie ses paroles. II faut se montrer à la hauteur de cette confiance. II y a toujours l'instant magique de la rencontre véritable entre l'artiste et son interprète. Vous êtes souvent présentée dans des circonstances de stress et de précipitation, avant de monter sur scène. Ils vous saluent poliment et font presque systématiquement une remarque sur le fait qu'ils n'ont pas grand-chose à dire, que leurs phrases seront courtes, etc. Puis, le moment fatidique arrive. On monte ensemble sur scène. L'artiste est grisé, parfois tendu, se lance dans une longue tirade et vous oublie. Vous le laissez s'exprimer librement, prendre ses marques d'orateur. Un moment de flottement dans la salle lui fait prendre conscience que (toute) l'assemblée ne comprend pas (tout) ce qu'il dit. II s'arrête soudain et se retourne vers vous d'un air confus. Vous lui adressez un sourire confiant et faites de votre mieux pour dire dans la langue de l'autre, de cette assemblée étrangère et bienveillante, ce qui vient d'être dit : mêmes données, mêmes affects, même implicite, même style. Et l'artiste vous découvre. II découvre plutôt qu'il a été écouté, entendu, avec précision, avec respect. Cela installe un triangle de communication passionnant, une polyphonie harmonieuse où l'interprète occupe son rôle d'intermédiaire. L'orateur se sent compris, le public sent qu'il comprend.

- Vous est-il arrivé de vous trouver dans des situations difficiles ou désagréables en tant qu'interprète dans le monde du cinéma, dues à la tension qui peut parfois régner dans ce milieu?

J'ai vécu une expérience surprenante lors de la dernière édition du Festival de Cannes. Un acteur américain était reçu en grande pompe par la Quinzaine des Réalisateurs. II est monté sur scène, en retard comme il se doit, et s'est emparé du temps imparti pour la présentation du film dont il était la star invitée pour se livrer à un véritable one-man-show. J'étais à ses côtés, chargée de traduire son discours, comme à l'accoutumée, sans rien connaître de ses intentions par avance, comme à l'accoutumée. C'était un acteur comique, grand spécialiste de grimaces et de farces en tout genre. La bienséance m'obligeait à arborer un sourire sans doute 
quelque peu figé, puisque, pendant ce temps - dix bonnes minutes -, mon cerveau s'activait pour tenter de fabriquer au fur et à mesure des équivalents à ses blagues et ses anecdotes truffées de jeux de mots, d'allusions à double sens, de sous-entendus faisant appel à un implicite proprement impénétrable pour quiconque ne connaissait pas sa filmographie par cœur... un réel supplice qui allait s'achever lorsqu'il a finalement remercié le public qui l'a salué par un tonnerre d'applaudissements. Enfin, je pouvais saisir le microphone et prononcer le premier mot qui interrompt les acclamations et me délivrer ainsi de cette pression accumulée en moi, me défaire de ce message qui me pesait tant, en le remettant à qui de droit. Mais à ce moment-là, le distributeur du film a soudain fait un signe depuis la salle vers la scène : le temps de présentation s'était écoulé, la star devait repartir sur le champ et le film devait être lancé ! Je suis descendue de scène dans un état physique pour moi inédit. Je me sentais extrêmement tendue, comme un volcan dont on aurait empêché l'éruption. Pendant les heures qui ont suivi, je me sentais comme préoccupée, ayant le plus grand mal à me concentrer sur un autre sujet, une autre conversation. Le soir, un autre film suivait. J'ai laissé de côté cet étrange épisode pour faire de la place dans mon esprit pour une nouvelle tâche d'interprétation. Mais je me souviens que la nuit, lorsque la tension de la longue journée de travail était retombée, j'ai de nouveau senti circuler dans ma mémoire les images, les tournures, les motsclés de ce discours déjà lointain qui refusait de s'estomper. J'étais presque tentée de le formuler à haute voix dans ma chambre d'hôtel, simplement pour m'en libérer et pouvoir dormir en toute quiétude! Au-delà de l'anecdote, j'ai eu là la preuve empirique que la phase la plus active, la plus intense de cet exercice d'interprétation consécutive est incontestablement la phase silencieuse, ou l'autre ne vous voit, ni ne vous entend travailler. C'est lors de cette étape que votre esprit perçoit, décortique, analyse, reformule, retient, se gonfle du message reçu. La phase de prise de parole ne consiste qu'à le laisser prendre son envol.

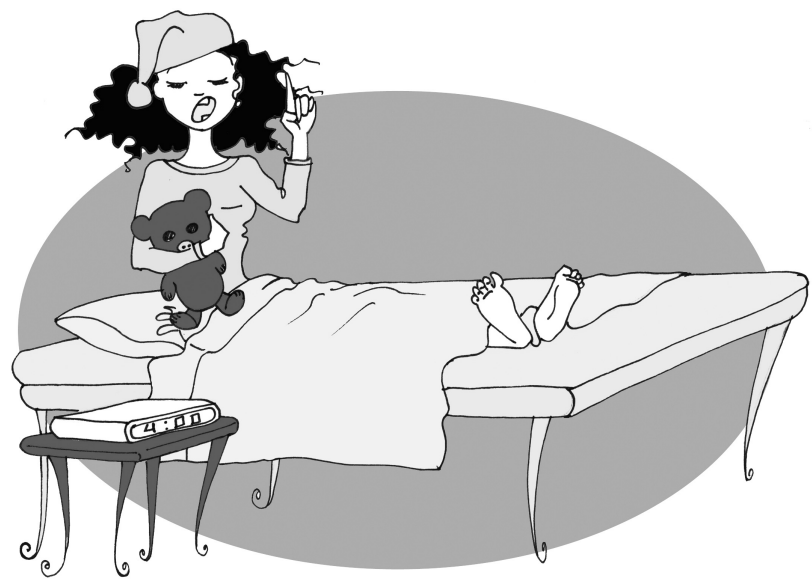

Illustration : Marlène Junius, http://alotoftralala.over-blog.com 
Pour ce qui est du milieu, je suis très heureuse d'y avoir fait mon entrée assez tard, et d'avoir eu d'autres expériences professionnelles auparavant. J'ai commencé à trente-cinq ans et je ne sais pas si avec dix ans de moins j'aurais su appréhender ce métier de façon sereine. Vous êtes très exposée. On vous voit dans les grands festivals, aux côtés de célébrités, on vous reconnaît, on vous interpelle et pourtant ce n'est jamais de vous qu'il est question! Personnellement, cela me convient parfaitement. Je n'aurais pas aimé être comédienne ou actrice, interprète en mon nom propre. J'aime cette ambiguité qui vous permet de dire " je " devant mille personnes sans jamais parler de vous. Vous ne perdez jamais de vue que dans ce triangle de communication, il y a deux interlocuteurs. L'axe à privilégier est celui qui les lie. Comme dans tout ménage à trois, il faut être très vigilant pour n'exclure personne! Ne pas jouer de la complicité naturelle qui s'établit entre l'artiste et vous. Ne pas oublier que celui qui vous écoute, à vos côtés, est l'auteur unique des propos que votre voix porte. Vous n'êtes qu'un intrus, qui doit rester en retrait, jusque dans sa posture. Tous vos efforts, toutes vos qualités (mais aussi vos défauts !) sont à verser au crédit de l'artiste qui est sur scène. Si vous faites une traduction pertinente de ses propos, si vous faites preuve d'humour ou de finesse, c'est lui qui en tire le bénéfice. Votre esprit, votre souffle, votre voix, sont au service du discours de l'autre. Je trouve cela magnifique ! II y a donc de nouveau ici un équilibre très délicat à trouver entre la présence et la discrétion. Vous êtes omniprésent et idéalement transparent. Pour faire ce métier, il ne faut craindre ni la lumière ni l'ombre.

- Vous venez de donner une clé essentielle : pour que l'interprète fasse bien son travail, il lui faut garder son sang-froid et maîtriser son stress. N'étant pas au premier plan, il n'y a pas de raison logiquement de subir la pression, même dans des situations très délicates...

Il faut relativiser l'importance de l'enjeu. Le trac ne s'explique pas, certaines personnes l'ont et d'autres non. Mais tout de même, je suppose que si j'étais à l'ONU et j'avais à traduire des propos dont dépend le sort de la planète, j'aurais des angoisses. Dans mon cas, je sais que personne ne mourra s'il y a une approximation dans ma traduction. Je n'oublie jamais que dans la vie, y compris dans la mienne, il y a des choses infiniment plus importantes que la présentation d'un film. Mais je crains qu'au festival de Cannes, mon attitude soit peu partagée. Tout le monde se sent investi d'une mission très importante. Cela dit, je fais mon travail très sérieusement. Je refuse simplement de me prendre au sérieux. Je suis très consciencieuse dans ma préparation. La condition absolue de ma détente au moment où je me jette à l'eau, c'est d'être très bien préparée. II y a des personnes qui font cela avec une certaine désinvolture, qui ne voient pas les films par exemple. Aller parler d'un film que l'on n'a pas vu, je trouve cela malhonnête, ou simplement dommage. Je prends un grand plaisir dans la phase préparatoire. Je m'enrichis et m'amuse beaucoup dans cette anticipation, cette immersion dans l'univers d'un artiste. Par conséquent, ce qui rend votre présence dans ce rôle légitime, ce n'est pas seulement vos compétences linguistiques mais ce travail de préparation. C'est cela qui fonde ce rapport de confiance qui me paraît si essentiel. Ces artistes voient que vous les 
connaissez, que vous avez su regarder leurs films, que vous vous êtes documentée sur leur œuvre. II peut arriver que je me retrouve à traduire quelqu'un sans avoir pu préparer. Je m'en sors toujours mais sans fierté. Cela me rappelle trop les commentaires sur les carnets de notes : "Réussit sans effort ». La seule chose qui peut rendre ce moment tendu, c'est le manque de préparation.

- Et la déformation professionnelle ? Avez-vous le sentiment d'interpréter en dehors des moments où vous travaillez?

J'ai le souvenir d'avoir appris les langues de cette façon-là. Je n'ai pas appris les langues pour devenir interprète, j'ai fait de l'interprétation pour apprendre les langues! Je veux dire que je les ai apprises sans résider dans les pays en question. Je me souviens quand j'étais lycéenne, je prenais le métro, j'entendais les gens parler autour de moi et j'essayais de traduire mentalement leurs propos en anglais, en espagnol, avec moins de bonheur en allemand ou en turc... C'est le conseil que j'ai toujours donné à mes étudiants. Apprendre une langue, ce n'est pas le travail d'une heure par jour. Si vous n'êtes pas obsédé par une langue, vous ne vous l'appropriez pas. II faut qu'elle soit quelque part dans votre esprit. Cela ne veut pas dire faire des exercices de grammaire ou apprendre les verbes irréguliers en permanence, mais y penser. Et penser, pour moi, c'est travailler. Peut-être parce que j'ai grandi en deux langues. Penser, pour moi, c'était interpréter. Je me demandais constamment quelles différentes formes les idées qui me traversaient l'esprit prendraient en fonction des langues. Ce mécanisme consistant à se dégager de la langue pour accéder à l'idée, puis transposer l'idée dans l'autre langue occupait constamment mon esprit. Un Monsieur Jourdain de l'interprétation, en somme !

- Vous passez donc à une nouvelle définition de l'art de l'interprète, très intéressante. L'interprète comme "reformuleur "...

C'est absolument ainsi que j'envisage mon travail. Une douce plaisanterie qui revient souvent dans la bouche des personnes que je traduis est qu'elles me demandent de les traduire aussi dans leur propre langue, puisqu'elles trouvent (quand elles comprennent la langue cible) que ma formulation est meilleure que la leur. Mais cela me semble évident. Interpréter, c'est donner une seconde chance à une idée. Leur expression est spontanée, brute. La mienne a une marge - certes limitée - de réorganisation de la pensée. Mais nous touchons là à l'éternel débat de la fidélité en traduction. II s'agit de s'emparer de toute la force, la vérité, la spontanéité d'un discours mais de la livrer sous une nouvelle forme. Comment ne pas saisir cette chance pour aller plus directement au cœur de ce que la personne cherche à dire ? Être fidèle, n'est-ce pas aussi donner ce que l'on a de meilleur? Par exemple, souvent, à la fin d'une prise de parole, les orateurs font une petite remarque qui rehausse le sens de ce qu'ils viennent de dire. II est donc fréquent que dans ma traduction, cette remarque apparaisse plutôt au début. La prise de décision doit être immédiate, intuitive. II s'agit de rendre le discours plus cohérent, plus juste, plus touchant, dans l'instant. 
C'est précisément le travail d'un monteur au cinéma. Une matière construite mais brute lui est livrée, il la réorganise, l'élague, lui insuffle un rythme, une dynamique qui la rende viable. Peutêtre de façon inconsciente, les cinéastes se sentent à l'aise dans ce partage des tâches, y compris lorsque leur film est achevé. Bien que je prenne un plaisir infini à traduire les propos d'écrivains ou de plasticiens, je crois que ma démarche est profondément marquée par le processus créatif du cinéma. Serge Daney parle des passeurs en cinéma. C'est vraiment la meilleure définition de ma perception du travail que je fais auprès des cinéastes. On me confie le trésor de la dernière étape de la réalisation d'un film et je le transmets à son destinataire ultime, le public.

- Vous avez travaillé avec de nombreuses personnalités du cinéma. Traduire leurs propos entraîne-t-il nécessairement des affinités avec elles?

L'un des grands avantages de ce métier a été d'élargir mon horizon de cinéphile. Je suis parfois amenée à me plonger dans l'univers de cinéastes dont je n'apprécie pas le travail a priori. II est intéressant de voir que, dans une certaine mesure, à l'instant où je traduis les propos d'une personne, je ne peux me départir d'une certaine empathie. Précisément, parce qu'il s'agit en règle générale, d'un discours incarné, personnel, empreint de conviction. Par conséquent, le contexte importe beaucoup. Lorsque je suis dans des festivals tels que Cannes ou Deauville et que je traduis des conférences de presse, je prends ma tâche comme une mission ponctuelle. Je laisse ma propre subjectivité de côté (dans la mesure du possible) et mets toute ma compétence de communication au service de cette personne. À la fin de la journée, il y a toutes les chances pour que cette incursion dans le monde et le discours de l'artiste en question (cinéaste ou acteur) soit close. Or, il arrive que je travaille de façon plus soutenue avec un auteur, par exemple pour une rétrospective consacrée à son œuvre. C'est la mission qui m'a été confiée par le Centre Pompidou, auprès de cinéastes tels que Guy Maddin, Werner Herzog ou Abbas Kiarostami. Dans ce cas, le travail est de longue haleine. Je dois étudier de façon bien plus approfondie le travail mais aussi l'univers artistique et intellectuel de ces auteurs et me livrer à leurs côtés à des exercices plus variés (entretiens, leçons de cinéma, débats, événements mondains...). Cela crée indéniablement une autre nature de relations, où vous devenez une sorte de "seconde voix " pour cet artiste, vous en arrivez à l'entendre penser. II s'établit alors un véritable échange, qui peut se prolonger au-delà de cette expérience ponctuelle. Cela a été particulièrement le cas avec Abbas Kiarostami, puisque depuis 2007, je suis devenue sa collaboratrice dans des domaines aussi variés que la mise en scène d'opéra, l'écriture de scénario ou la direction d'acteurs pour son dernier film. II est clair que toutes ces activités ne peuvent s'inscrire dans le strict cadre du métier d'interprète, même s'il s'agit toujours de recueillir la pensée de l'autre, la mettre en forme dans d'autres langues et la transmettre à ses divers destinataires. 
- Nous parlions précédemment des affinités avec les personnes, et qu'en est-il des affinités avec vos langues de travail ? Avez-vous plus de facilité dans l'une d'entre elles?

L'anglais et l'espagnol sont mes langues de formation universitaire. Le français est la langue que je considère comme ma langue première, celle de ma formation intellectuelle, puisque mon éducation primaire a été bilingue mais qu'à partir du collège, j'ai été scolarisée à Paris. Cependant, dès mon arrivée en France à l'âge de douze ans, j'étais consciente que si je me contentais des conversations familiales, je ne développerais pas ma pratique de ma langue maternelle de façon satisfaisante. J'avais tout juste commencé à prendre goût à la littérature et à la poésie persanes et je sentais que quelque chose m'échapperait si je me contentais d'une relation nourricière, vernaculaire à cette langue. J'ai donc lu, écrit, seule, pendant toutes ces années. Je ne suis jamais retournée en Iran depuis. J'ai étudié cette langue et cette poésie en dilettante passionnée, par à-coups. Lorsque j'ai commencé à travailler pour le cinéma, j'annonçais d'emblée mes quatre langues de travail même si j'étais le plus souvent sollicitée pour l'anglais. Je travaille plus rarement en espagnol, peut-être simplement car les " clients " ont tendance à vous associer à une langue de travail. Pour le persan, la question était encore plus délicate. Étant donné qu'il s'agit d'une langue rare, que les films iraniens ont des sorties plus confidentielles, donc des budgets moindres, l'on avait le plus souvent recours à des persanophones non professionnels pour les tâches de traduction (sous-titrage ou interprétariat). Pour ma part, je n'ai jamais entrepris de démarche spontanée pour proposer mes services et n'ai donc pas travaillé en persan dans un premier temps. Ma collaboration avec Kiarostami a changé la donne. Des professionnels du cinéma voyant mon travail avec lui m'ont proposé d'être l'interprète d'autres cinéastes ou de sous-titrer leurs films. Et il est très intéressant d'observer qu'il demeure parfois une résistance face à la pratique de tarifs professionnels concernant le persan! Cela vient tout simplement de la mauvaise habitude prise avec le travail amateur. Je me retrouve donc souvent chargée d'une mission pédagogique à l'encontre de clients à qui il faut démontrer la valeur d'un travail professionnel. Certains d'entre eux le comprennent d'autant plus aisément qu'ils ont eu à pâtir des conséquences de traductions médiocres de la part de non professionnels. Mais il arrive que le message passe difficilement étant donné la conjonction de deux critères : le domaine du cinéma, où l'on pourrait croire qu'après tout, il n'est pas difficile de comprendre "à peu près " ce que dit un réalisateur, et que n'importe quel stagiaire connaissant vaguement sa langue peut le traduire ; doublé de l'argument économique dont on use pour demander au traducteur de faire un effort pour un film iranien, puisque, comme chacun le sait, il ne se vendra pas aussi bien qu'un film des studios hollywoodiens. Mais demande-t-on aux laboratoires de tirer des copies à moindre prix pour ce même film?

$\|$ y a une tendance, de la part des commanditaires, à vous cataloguer selon une langue, voire une cinématographie. C'est donc à vous de ne pas vous laisser cantonner à un domaine à travers le choix des missions que vous acceptez. Bien sûr, dans les festivals, le fait d'avoir 
quatre langues de travail est un atout précieux. II m'est déjà arrivé d'être sur scène avec des locuteurs des trois langues - anglais, espagnol, persan - face à une salle francophone et de devoir interpréter dans toutes les combinaisons possibles! Mais globalement, l'anglais domine largement dans les demandes qui me sont adressées.

- Que signifie pour vous travailler avec votre langue maternelle?

Vous l'avez compris, j'ai un rapport très affectif au persan. Ce qui me reste du pays de mon enfance, c'est sa poésie, son esprit, sa saveur, la finesse et la richesse de sa langue. Ce rapport est nourri par le travail que je fais avec Kiarostami. Ses films m'ont permis pendant des années de me plonger dans cette beauté, de garder un lien fort avec des paysages, des êtres, des sons qui étaient pour moi perdus. Travailler avec lui est un cadeau, inattendu et évident. Son langage cinématographique me touche autant que son regard de photographe, que sa plume de poète. Et sa parole témoigne d'une pensée forte, portée par une poésie immédiate, permanente. Je travaille avec lui vers le français et vers l'anglais et je suis très touchée de voir que ses interlocuteurs reconnaissent à travers la traduction l'unicité de son style, de son dire.

- Pour vous ce sont donc des conditions de travail idéales, qui vous plaisent parce que vous connaissez les personnes et pouvez pratiquement anticiper leur propos, et parallèlement interpréter dans l'instant avec des gens que vous ne connaissez pas du tout... Combiner ces deux choses c'est véritablement ce qui vous comble dans votre métier?

En tout cas, il est certain que ce métier me comble ! J'ai toujours préféré agir plutôt que rêver mais je crois que si on m'avait demandé de désigner le métier de mes rêves, j'aurais choisi celuici. Comme je vous disais, je suis quelqu'un d'assez obtus. J'ai deux passions : les langues et le cinéma. Que pouvais-je trouver de plus intéressant à faire ? Seulement, je n'ai pas rêvé de ce métier car il n'existait pas. Le bonheur est ce que l'on invente. Je n'ai jamais été convaincue lorsque l'on m'a dit que pour vivre, il fallait se conformer à des schémas préétablis. Je ne l'ai fait dans aucun domaine. Ce qui me comble également dans ce travail, c'est son aspect éphémère. Vous ne laissez pas de trace. Vous donnez tout dans l'instant et n'en tirez nul autre bénéfice que la grâce de l'instant. Pouvoir vivre de ces intuitions, de ces enthousiasmes, dire " je " à la place d'êtres d'exception, passer l'essentiel de son temps à écouter et à regarder, voir les visages des spectateurs s'épanouir à mesure que le sens leur est dévoilé. Je manque d'imagination pour concevoir un métier plus heureux. Pour l'instant. 